\title{
Adverse Drug Events and Associated Factors in Heart Failure Therapy Among the Very Elderly
}

\author{
Richard Sztramko, BHSc, MD, FRCPC ${ }^{1}$, Vicky Chau, MD $^{2}$, Roger Wong, MD, FRCPC ${ }^{1}$ \\ ${ }^{I}$ Division of Geriatric Medicine, Department of Medicine, University of British Columbia, Vancouver, BC; \\ ${ }^{2}$ Department of Medicine, University of British Columbia, Vancouver, BC, Canada
}

DOI: $10.5770 /$ cgj.v14i4.19

\begin{abstract}
\section{Introduction}

Heart failure (HF) is common in older adults and standard therapy involves the use of multiple medications. We assessed the nature, frequency, and factors associated with adverse drug events (ADEs) associated with standard HF therapy among older adults greater than 75 years of age. The efficacy and predictors of ADEs were assessed in this patient population, as well.
\end{abstract}

\section{Methods}

Systematic review using standardized databases including MEDLINE, Ageline, and CINAHL from January 1st 1988 to January 1st, 2010 and references from published literature. Randomized trials and studies with observational, cohort, and cross-sectional design were included. Two investigators independently selected the studies and extracted the data $(\mathrm{kappa}=0.86)$.

\section{Results}

Twenty-five studies were identified. ADEs were reported in 13/23 (57\%) studies. Syncope, bradycardia, and hypotension as a result of beta blockers occurred in greater frequency compared to younger populations. Spironolactone therapy resulted in increased rates of hyperkalemia, acute renal failure, and medication discontinuation. Factors associated with ADEs included advanced age, poor left ventricular function, and increasing New York Heart Association Class. Efficacy of beta blockers and ACE inhibitors appears to extend to the elderly population, but the magnitude of effect size is unclear. Very few studies reported associations between ADE and patients' comorbidities (4/13 studies, $31 \%$ ) or functional status (3/13 studies, $23 \%)$.

\section{Conclusion}

ADEs in CHF therapy among the very elderly occurred at a greater frequency, but were generally poorly characterized in the literature despite a relatively common occurrence. Further studies are warranted.

Keywords: congestive heart failure, heart failure, adverse drug events, medication side effects, elderly, frailty, medical comorbidities

\section{INTRODUCTION}

Heart Failure (HF) is an extremely common condition in the elderly. In fact, the median age of onset for HF is 75 , with half of the patients with the condition being over $800^{(1,2,3)}$ Many medical therapies have been shown to reduce the morbidity and mortality associated with systolic HF; however, the average age of study participants has not been representative of the majority of the patients with the condition. ${ }^{(4,5,6)}$ Much of the evidence used to justify the benefit of therapies has been extrapolated to the elderly population in whom cardiovascular physiology is altered, pharmacokinetics and dynamics are different, and drug interactions and susceptibility to side effects are increased. . $^{(7,8,9)}$

It was our hypothesis that elderly patients would experience adverse drug events (ADEs) at a higher rate than conventional trials have reported, and that concepts such as frailty and medical comorbidities have not been extensively evaluated to see if there are predictors of ADEs. A secondary hypothesis was that there would be a small amount of highquality data to support the current heart failure treatment paradigm in people over the age of 75 .

The purpose of this study was to quantify the degree of ADEs in the elderly population related to their HF therapy, understand their nature and, if it differs from the younger populations studies, establish whether the therapies are efficacious, and determine whether there are predictors of adverse ADEs related to the elderly population.

\section{METHODS}

A systematic review was performed using standardized databases including MEDLINE, AgeLine, CINAHL, and the Cochrane 
Reviews, from January, 1988, to January $1^{\text {st }}, 2010$. References from the published literature were also included. Keywords included: congestive heart failure, heart failure, adverse drug events, medication side effects, polypharmacy, elderly, frailty, and medical comorbidities. Meta-analyses, randomized controlled trials (RCTs), observational studies, and reviews were included. Only studies involving patients with a median or mean age of 75 years, or with a treatment arm that involved patients with a median or mean age of 75 years, were included. Medications reviewed included angiotensin-converting enzyme inhibitors, beta blockers, spironolactone, and digoxin.

Two reviewers (VC and MB) independently examined each study based on the prespecified inclusion criteria. Where there was disagreement, a third reviewer (RS) was included, and consensus was reached as to whether the article would be included or not. Data was independently extracted and examined by $\mathrm{VC}$ and $\mathrm{RS}$ targeting prespecified variables: medications used, adverse events measured, point prevalence of those events, hospitalizations, mortality, symptom control, comorbidities measures, functionality, and frailty. Variables of interest measured that were not prespecified were tolerability, undertreatment, or special study populations such as diabetics or patients with atrial fibrillation. A standardized data extraction form in Microsoft Excel format was used. A post-hoc analysis of study quality was performed on trials, as well as observational data. Studies were only included if they had Jadad scores of 2 for RCTs or Newcastle-Ottawa Quality Assessment scores of 6 or greater. ${ }^{(10,11)}$

Side effect rates from the observational data were pooled for comparison when possible and compared to rates noted in the randomized control trials. Data heterogeneity was controlled for using Cochran's Q statistic. The Cochran's Q statistic was not intended to be the statistical test of choice; however, given the large number of observational and cohort studies, it was felt to be the most appropriate, despite the inherent biases and lack of power to attain firm conclusions.

\section{RESULTS}

Our initial literature search yielded a total of 213 studies. After we reviewed the titles and abstract, a total of 25 papers met inclusion criteria and were selected for final analysis. (Figure 1) The kappa statistic was 0.86 for inter-rater reliability. More than half of the studies (13) represented heart failure patients with systolic dysfunction. While diastolic dysfunction is very prominent in the elderly, there were only two studies that investigated this population specifically. There were six papers that included patients with both systolic and diastolic dysfunction. The studies are summarized in Table 1.

\section{Adverse Drug Events}

\section{Beta Blockers}

A total of seven studies evaluated beta blocker use for heart failure in the elderly. ADE rates from the observational studies $^{(12,13,14,15,16,17)}$ were pooled and compared to randomized control trial data from the SENIORS ${ }^{(18)}$ and COPERNICUS ${ }^{(19)}$ trials. The SENIORS trial is a randomized control trial using nebivolol in the elderly population, with a mean age of 79. The COPERNICUS trial explicitly stated adverse event rates related to carvedilol in patients with a mean age of 63. It was used as a benchmark for the incidence of medication side effects in randomized control trials in the nonelderly population.

There were discrepancies in the incidence of adverse events between the elderly and nonelderly groups treated with beta blockers. Four percent (48/1101) of the review study cohort experienced bradycardia, as did $11 \%$ of the patients in the SENIORS trial. ${ }^{(18)}$ In comparison, only 2\% (22/1156) of the younger patients in the COPERNICUS trial did. ${ }^{(19)}$ The incidence of hypotension was 13\% (106/829) in the review cohort, 8\% (82/1067) in SENIORS, and only 2\% (22/1156) in COPERNICUS. There were similar rates of syncope with $17 \%(29 / 176)$ in the review cohort and 16\% (166/1067) in SENIORS, while again a large discrepancy with only $2 \%$ (19/1156) of patients experiencing these symptoms in COPERNICUS. (Table 2)

Many other adverse events were reported in a nonuniform manner and could, therefore, not be compared. These included: bronchospasm, shortness of breath, arrhythmias, worsening functional status, and gastrointestinal complaints.

The most common beta blockers studied in descending order were carvedilol, bisoprolol, and nebivolol. A mean follow-up period of 14.93 months was observed. Pooled tolerability in all seven studies was $65.57 \%$ (1811/2762). The tolerability of carvedilol in the COPERNICUS trial was $70 \%$. (19) The mean tolerated dose for carvedilol was $31.73 \mathrm{mg}$ daily. The mean dose for bisoprolol was $7.6 \mathrm{mg}$ daily, and the mean tolerated dose for nebivolol was $7.7 \mathrm{mg}$ daily.

\section{ACE Inhibitors}

Three studies reported ADEs in relation to ACE inhibitor use. $^{(20,21,22)}$ The first was a randomized, double-blind trial evaluating the use of quinapril in 36 diastolic heart failure patients. The other two studies looked at initiating chronic therapy, but specifically did not look at how those medications administered chronically resulted in adverse events, which was the focus of our study. The point prevalence of adverse events in the 36 patients treated with quinapril in the first study did not lend themselves to any comparisons due to the small numbers. No valuable comparisons came out of evaluating the ACE inhibitor studies.

\section{Spironolactone}

Three studies evaluated the use of spironolactone. ${ }^{(23,24,25)}$ Dinsdale et al. ${ }^{(23)}$ looked at the use of spironolactone in a retrospective case series of 64 patients hospitalized with heart failure at their institution, with a mean age of 85 . Spironolactone was prescribed in conjunction with an ACE 


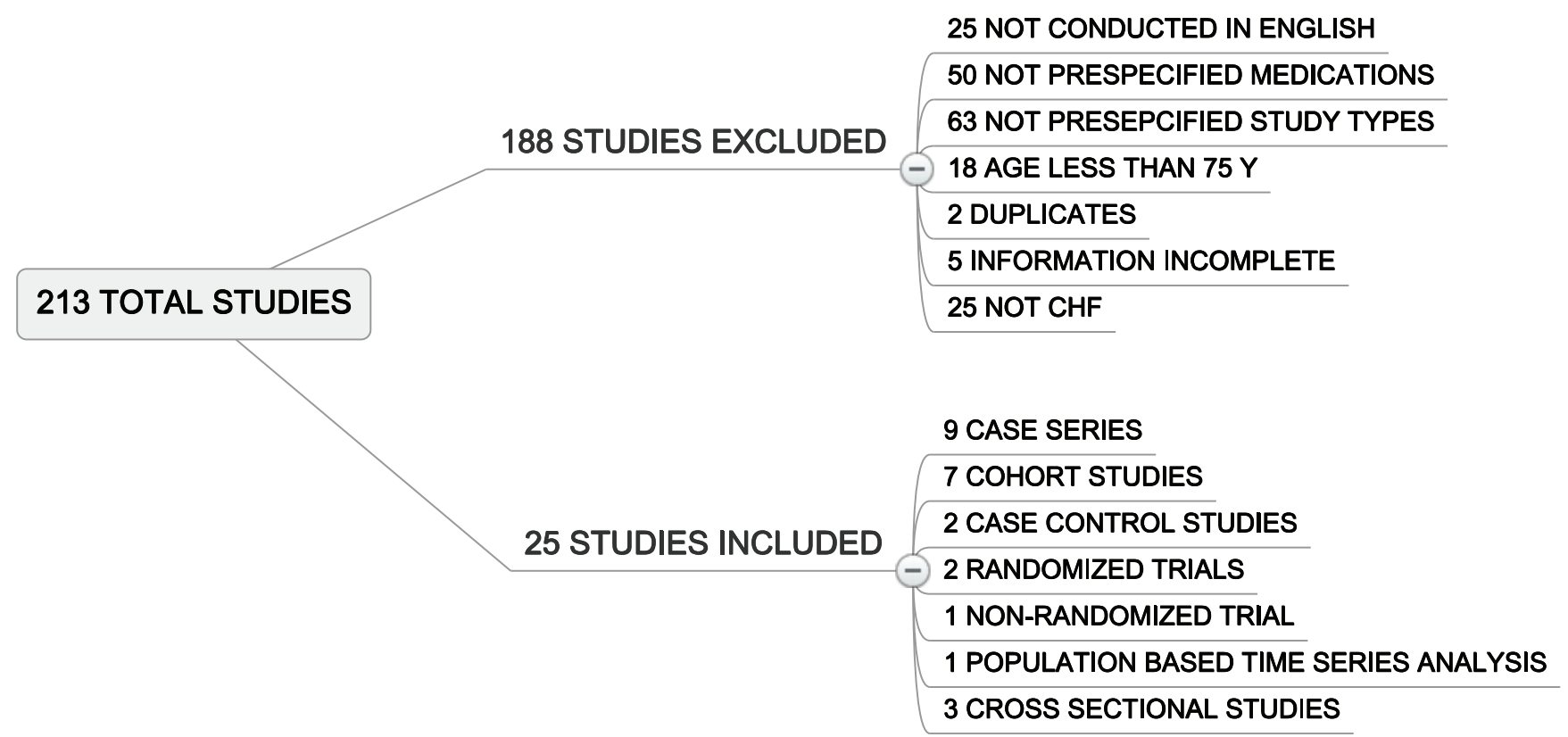

FIGURE 1. Reasons for exclusion

inhibitor. The rate of adverse drug events in this cohort was compared to the RALES study participants. The definition of an adverse drug event was uniform across both studies.

The rate of severe hyperkalemia in the Dinsdale study was between $11 \%$ for severe ( $>6.0 \mathrm{mmol} / \mathrm{L})$, and $36 \%$ for hyperkalemia in general $(>5.5 \mathrm{mmol})$. The rate of serious hyperkalemia in the RALES trial was only $2 \%$. Forty-three percent of patients in the Dinsdale study had to discontinue spironolactone because of acute renal failure, hyperkalemia, or postural hypotension. Only $8 \%$ of patients in the RALES trial discontinued the medication. ${ }^{(23,6)}$ (Table 3).

In a population-based time series analysis, Juurlink et $a l^{(24)}$ evaluated the rates of hyperkalemia-associated hospitalizations and death after the online publication of the RALES trial in 1999. They showed that as prescriptions increased from $34 / 1000$ to $149 / 1000$, hyperkalemia-related hospitalizations increased from $2.2 / 1000$ to $11 / 1000$, and hyperkalemiaassociated deaths increased from $0.3 / 1000$ to $2 / 1000$. The mean age of patients in this cohort was 78.5 years of age. The goal of this study was to elaborate on the differences between patients treated in randomized control trials with frequent follow-up and strict inclusion criteria, as compared to "reallife" patients, some of whom do not fit the specifications for receiving the medication. It is notable that patients experiencing the majority of the side effects are above 75 years of age.

Butler et al. ${ }^{(25)}$ looked at the addition of spironolactone to 18 systolic heart failure patients with a mean age of 80 years of age. Nine patients had a baseline serum creatinine above $150 \mathrm{mmol} / \mathrm{L}$, and nine patients had a serum creatinine below 150 with a mean of 89.6. The addition of spironolactone resulted in hyperkalemia in six patients, or $66 \%$ of patients with baseline serum creatinines above 150 , and only one patient with a normal serum creatinine. All potassium levels returned to normal when the dose of spironolactone was decreased to $12.5 \mathrm{mg}$ daily from 25 . No other serious side effects were found that required discontinuation of the medication.

\section{Digoxin}

Three studies looked at ADEs in relation to digoxin use. Carosella et al. ${ }^{(26)}$ looked at over 20,000 patients hospitalized for HF in Italy between 1988 and 1993. They found that although the mean prescribed dose of digoxin was lower in the elderly, age was an independent risk factor of ADEs. Other predictors of ADRs were daily dosage of digoxin $>0.25 \mathrm{mg}$, serum creatinine $>120 \mathrm{micrograms} / \mathrm{L}$, the number of drugs taken prior to admission, administration of amiodarone, propafenone, quinidine, or verapamil.

Misiaszek et al. ${ }^{(27)}$ looked specifically at the elderly in long-term care facilities and found that $33 \%$ of patients with HF were being prescribed digoxin. Most of these patients had atrial fibrillation. The authors found that $26 \%$ of these patients were at risk for adverse events because their serum concentrations were greater than 1.5 , or because they were being prescribed medications that increased their risk of ADEs.

\section{Comorbidities and Functional Status as a Predictor of ADEs}

Out of 24 studies, 16 measured medical comorbidities in some manner. Nine studies used a validated index, and six used a nonsystematic approach. The most commonly used validated models were the Charlson and Elixhauser comorbidities 


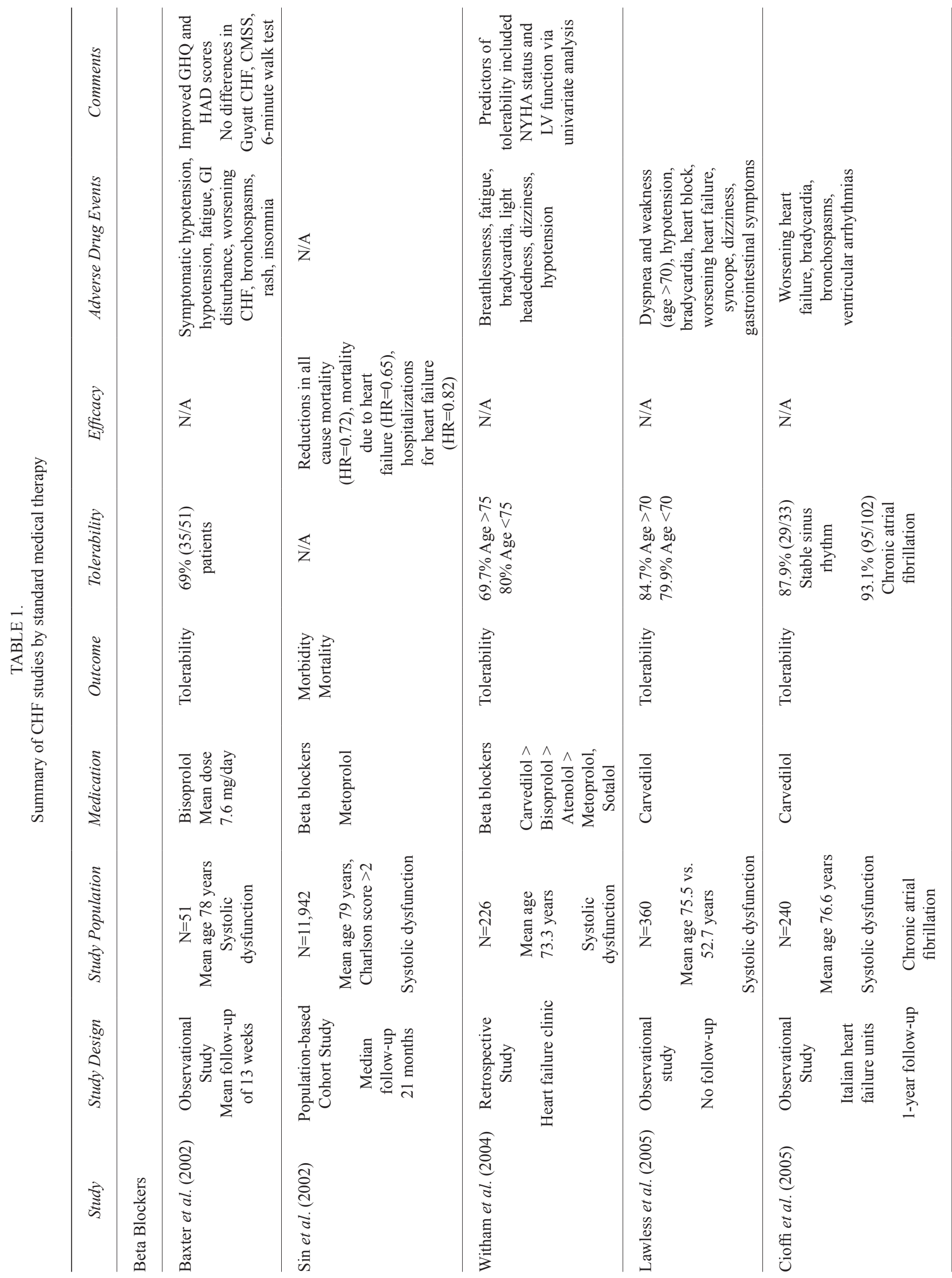


SZTRAMKO: ADVERSE DRUG EVENTS

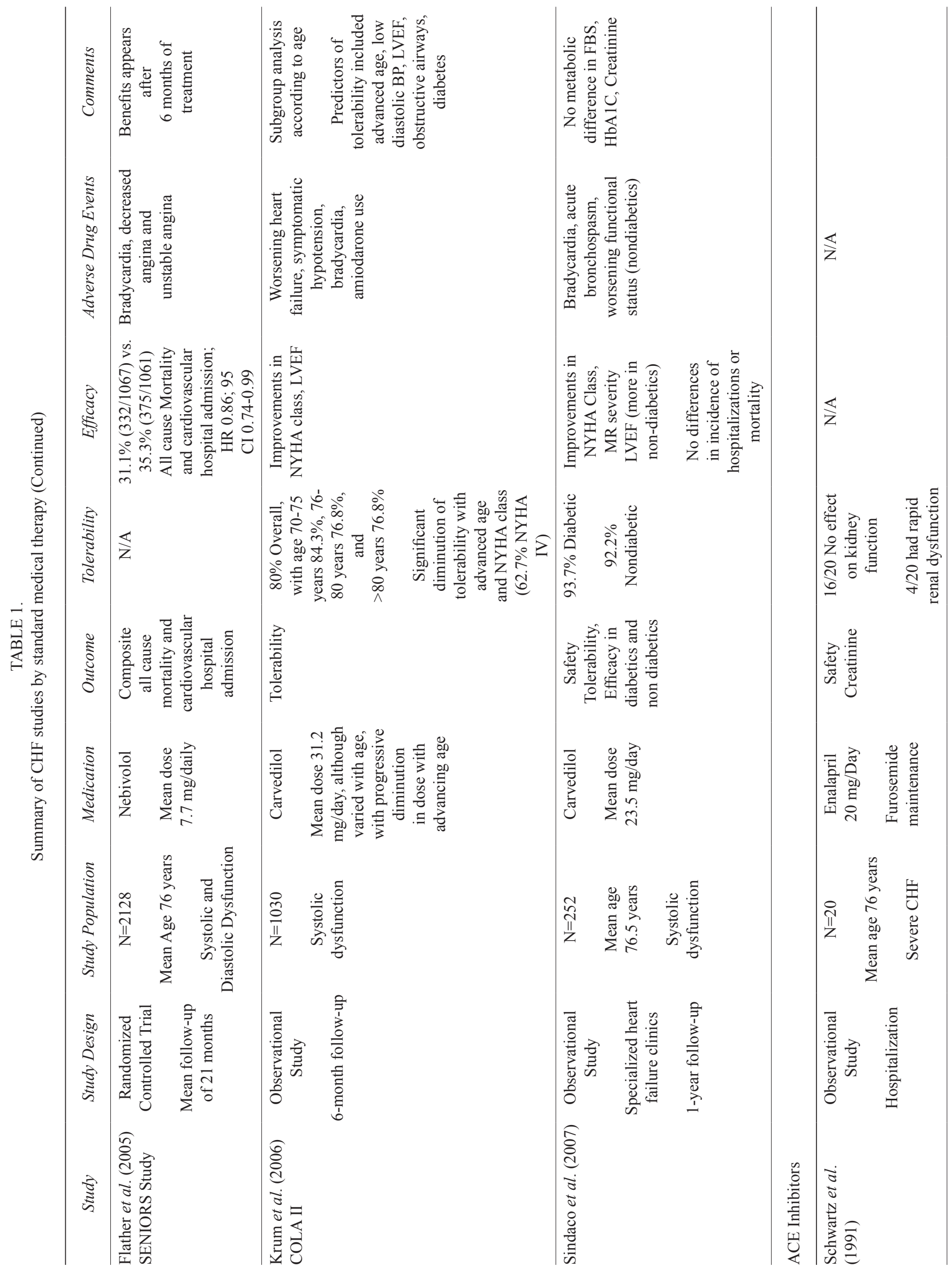

CANADIAN GERIATRICS JOURNAL, VOLUME 14, ISSUE 4, DECEMBER 2011 
SZTRAMKO: ADVERSE DRUG EVENTS

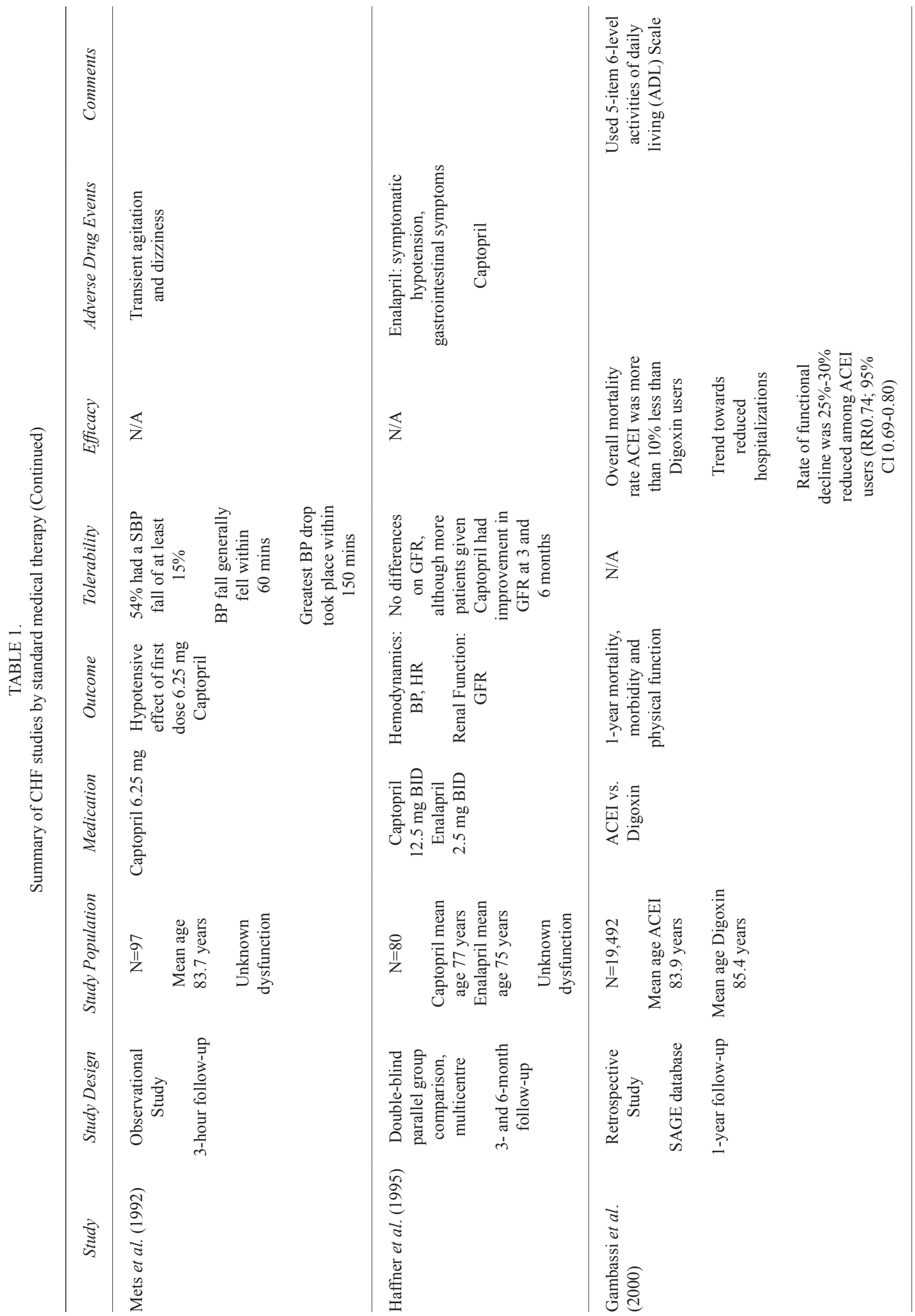




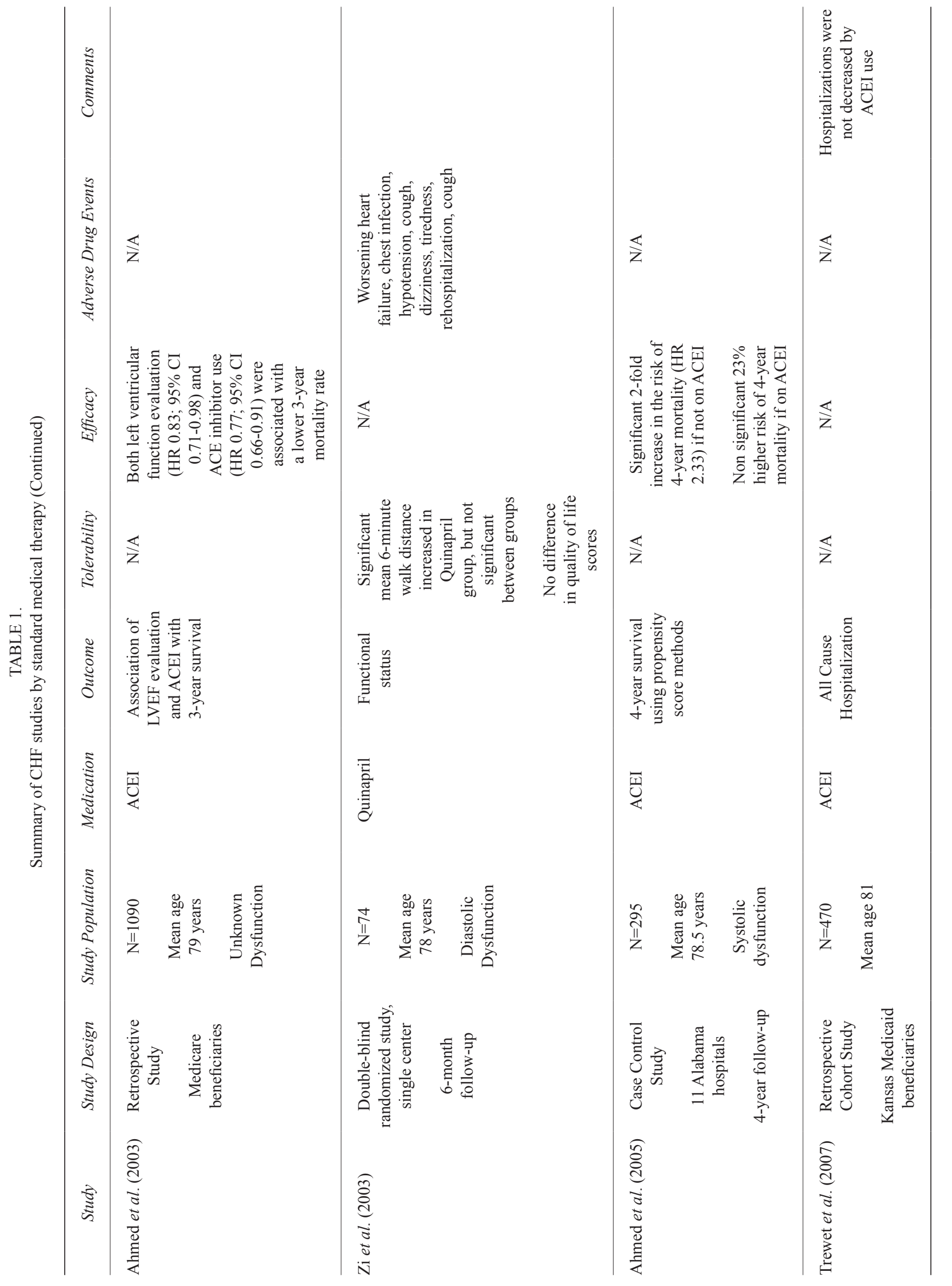

CANADIAN GERIATRICS JOURNAL, VOLUME 14, ISSUE 4, DECEMBER 2011 
SZTRAMKO: ADVERSE DRUG EVENTS
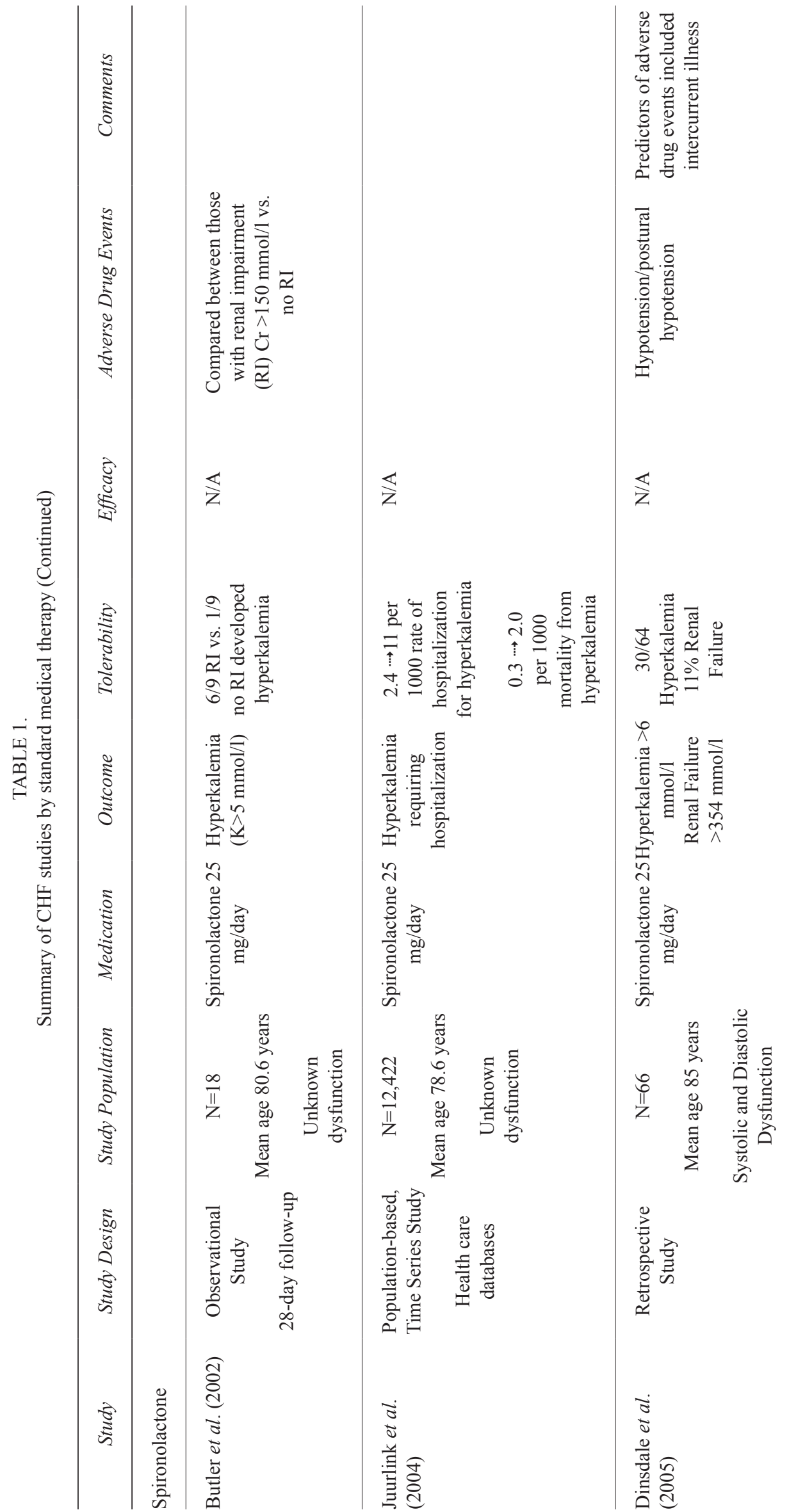

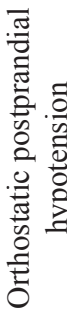

$\overleftrightarrow{\mathrm{Z}}$

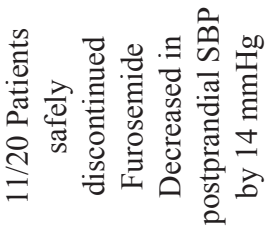

吾 .

필

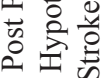

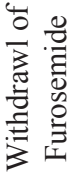

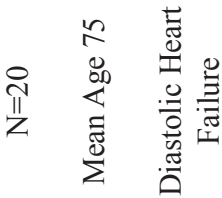

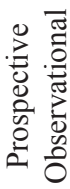

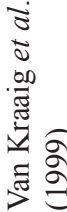


SZTRAMKO: ADVERSE DRUG EVENTS

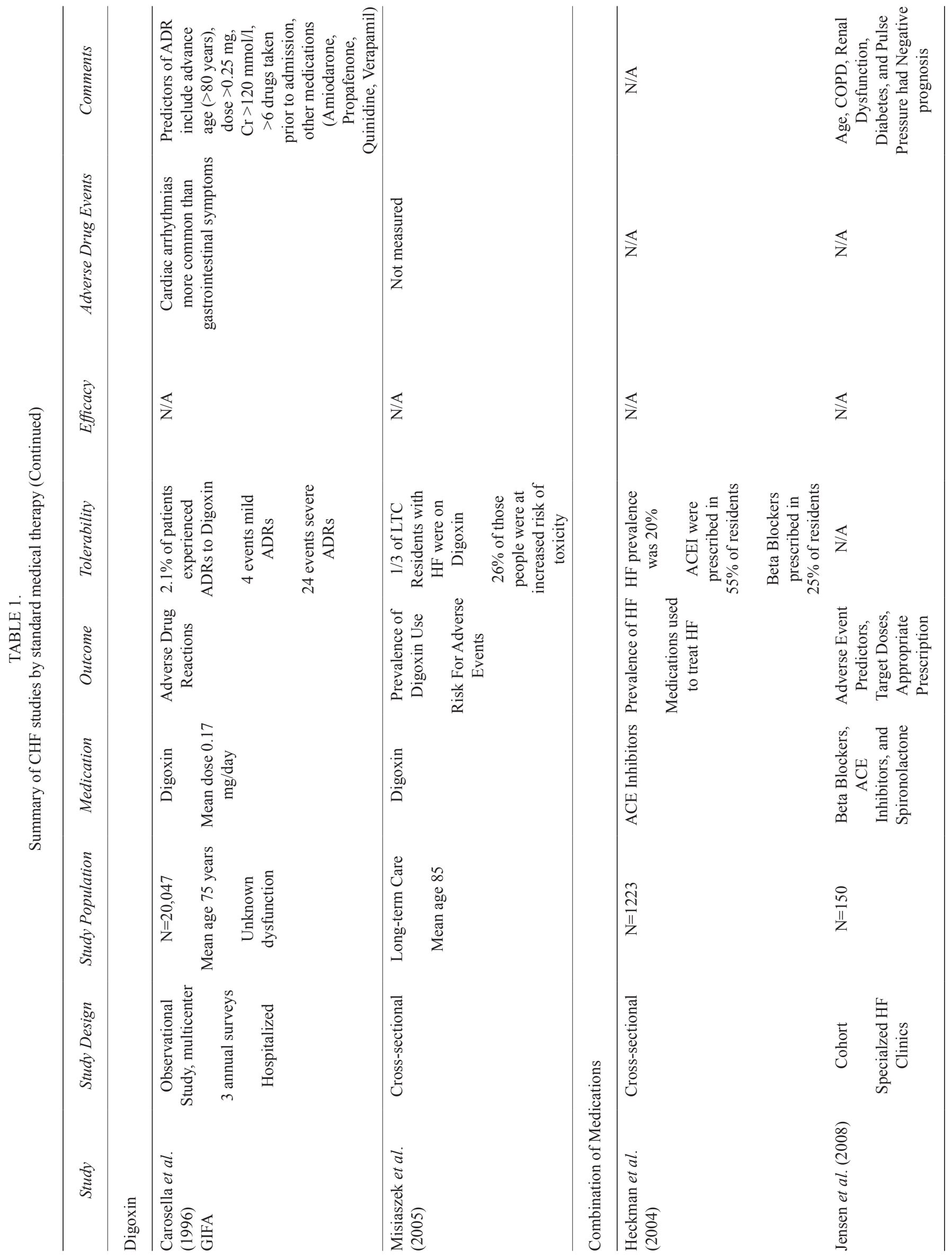

CANADIAN GERIATRICS JOURNAL, VOLUME 14, ISSUE 4, DECEMBER 2011 
TABLE 2.

Adverse drug events compared between observational, RCT data in those over 75 and RCT data in patients less than 75 years of age

\begin{tabular}{lccc}
\hline \multicolumn{1}{c}{ Side Effect } & $\begin{array}{c}\text { Pooled } \\
\text { Observational } \\
\text { Data } \\
\text { (Mean age }>75)\end{array}$ & $\begin{array}{c}\text { Seniors } \\
\text { Trial } \\
\text { (Mean } \\
\text { age } 79)\end{array}$ & $\begin{array}{c}\text { Copernicus Trial } \\
\text { (Mean Age 63) }\end{array}$ \\
\hline Bradycardia & $4 \%(48 / 1101)$ & $\begin{array}{c}11 \% \\
(118 / 1067)\end{array}$ & $1.5 \%(17 / 1156)$ \\
Hypotension & $13 \%(106 / 829)$ & $8 \%$ & $1.9 \%(22 / 1156)$ \\
& & $(82 / 1067)$ & \\
Worsening HF & $5 \%(44 / 912)$ & $\begin{array}{c}24 \% \\
(256 / 1067)\end{array}$ & Not Reported \\
& & $7 \%$ & Not Reported \\
Weakness/Fatigue & $19 \%(51 / 222)$ & $\begin{array}{c}7 \% \\
(72 / 1067)\end{array}$ & \\
& & $16 \%$ & $1.6 \%(19 / 1156)$ \\
Syncope & $17 \%(29 / 171)$ & $(166 / 1067)$ & \\
\hline
\end{tabular}

TABLE 3.

Adverse drug events related to sprionolactone therapy

\begin{tabular}{lccc}
\hline \multicolumn{1}{c}{ Side Effect } & $\begin{array}{c}\text { Dinsdale et al. } \\
\mathrm{N}=64\end{array}$ & $\begin{array}{c}\text { Butler et al. } \\
\mathrm{N}=18\end{array}$ & $\begin{array}{c}\text { RALES } \\
\mathrm{N}=1663\end{array}$ \\
\hline $\begin{array}{l}\text { Hyperkalemia }>5.5 \\
\text { mmol/L }\end{array}$ & $36 \%$ & $33 \%$ & $2 \%$ \\
Acute Kidney Injury & $37.5 \%$ & 0 & Not Reported \\
Discontinuation & $34 \%$ & 0 & $8 \%$ \\
\hline
\end{tabular}

measures. Despite the fact that the authors seemed very cognizant of the fact that medical comorbidities might impact health outcomes, only three studies attempted to correlate comorbidities with impact.

Krum et $a l .^{(13)}$ published a prospective observational study on the tolerability of carvedilol in a cohort of 1030 elderly patients with systolic heart failure. Key measures that may predict tolerability were measured at baseline, and the ability to remain on $6.25 \mathrm{mg}$ twice daily at six months were measured. Factors associated with decreased medication tolerance were: advanced age, obstructive airways disease, advanced NYHA class, and hospitalization in the previous six months. Factors that increased tolerability were higher ejection fractions, increased systolic and diastolic blood pressures, and the presence of diabetes. With all things considered, the beta blocker was tolerated in $80 \%$ of patients overall in patients that were greater than 75 years of age.
A study by Witham et $a l .^{(17)}$ compared the tolerability of carvedilol in 110 patients with a mean age of 80 years to 116 patients with a mean age of 65 years of age. This study did agree with higher NYHA class and lower LV function as predictors of failing a trial of beta blocker therapy. The data of this study did conflict with the previous study in that age, and a diagnosis of obstructive airways disease did not predict treatment failure.

This study was specifically looking at the tolerability of beta blocker therapy in the elderly, and found no difference in tolerability between the patients less than 75 when compared to patients greater than 75 years of age. It used the Charlson Comorbidity Index, and found that the older patients did have a higher rate of symptomatic heart failure, musculoskeletal complaints, hypertension, and stroke. Despite these comorbidities being present, none of these predicted beta blocker failure from side effects, except for those already mentioned.

In the retrospective study performed by Dinsdale et al., ${ }^{(23)}$ medical comorbidities were not measured in a validated scale, but the authors did attempt to see whether comorbidities were predictors of adverse events, specifically hyperkalemia and acute renal failure. No significant relationship was found between age, baseline creatinine, ACE inhibitor dose, NYHA class, diabetes, intensity of monitoring, or number of medications, and the aforementioned adverse events. Predictors of ADEs are summarized in Table 4.

Functional status was measured in three trials. ${ }^{(22,28,29)}$ The impact of functional status on adverse drug events and overall tolerability was not measured.

\section{Efficacy}

\section{Beta Blockers}

The SENIORS trial was a prospective, randomized control trial to evaluate the use of nebivolol in elderly patients with systolic HF. One thousand and sixty-seven (1067) patients were randomized to receive $10 \mathrm{mg}$ daily of nebivolol, and 1061 to placebo, and were followed for 21 months. The mean age of the study participants was 76 , with $37 \%$ being female. The mean ejection fraction was $36 \%$, with $35 \%$ of participants having an ejection fraction of less than $35 \%$. The primary combined outcome of hospitalization or mortality occurred in $31.1 \%$ of patients treated with nebivolol and $35.3 \%$ in the placebo group, with a hazard ratio of $0.86(0.74-0.99)$. Mortality occurred in $15.8 \%$ on nebivolol and $18.1 \%$ in the placebo group, with a nonsignificant hazard ratio of 0.88 $(0.71-1.08 ; p=0.21){ }^{(18)}$

The hazard ratio for mortality when specified by age group revealed a statistically significant reduction of 0.79 (0.63-0.98) in patients less than 75.2 years of age, and a nonsignificant hazard ratio of $0.92(0.75-1.12)$ for those aged greater than 75.2 years of age. ${ }^{(18)}$ Nebivolol appears to have a significant impact on mortality and hospitalizations in the elderly, but its benefits appear to be disproportionately greater in younger patients. 
TABLE 4.

Predictors of adverse events/tolerability

\begin{tabular}{|c|c|c|c|c|}
\hline Study & $\begin{array}{c}\text { Krum et al. } 2006 \\
\text { Carvedilol }\end{array}$ & $\begin{array}{c}\text { Dinsdale et al. } 2005 \\
\text { Spironolactone }\end{array}$ & $\begin{array}{c}\text { Witham et al. } 2005 \\
\text { Beta Blockers }\end{array}$ & $\begin{array}{c}\text { Carosella et al. } 1996 \\
\text { Digoxin }\end{array}$ \\
\hline \multicolumn{5}{|l|}{ PREDICTOR } \\
\hline Older Age & Increased $A D E$ & No Effect & No Effect & Increased $A D E$ \\
\hline Advanced NYHA & Increased $A D E$ & No Effect & Increased $A D E$ & Not Studied \\
\hline Increased LVEF & Decreased ADE & No Effect & Decreased ADE & Not Studied \\
\hline Increased DBP & Decreased $A D E$ & Not Studied & Not Studied & Not Studied \\
\hline Increased SBP & $\begin{array}{c}\text { Decreased } \\
\text { ADE }\end{array}$ & Not Studied & Not Studied & Not Studied \\
\hline Presence of COPD & Increased ADE & Not Studied & No Effect & Not Studied \\
\hline Intercurrent Illness & Not Studied & Increased $A D E$ & Not studied & Not Studied \\
\hline $\begin{array}{l}\text { Higher Diuretic } \\
\text { Dose }\end{array}$ & Not Studied & Not Studied & No Effect & Not Studied \\
\hline $\begin{array}{l}\text { Multiple } \\
\text { Medications }\end{array}$ & Not Studied & No Effect & Not Studied & Increased ADE \\
\hline $\begin{array}{l}\text { Digoxin Dose } \\
\text { Greater than } 0.25 \mathrm{mg}\end{array}$ & Not Studied & Not Studied & Not Studied & $\begin{array}{c}\text { Increased } \\
\text { ADE }\end{array}$ \\
\hline $\begin{array}{l}\text { Creatinine } \\
>120 \mathrm{mmol} / \mathrm{L}\end{array}$ & Not Studied & Not Studied & Not Studied & $\begin{array}{c}\text { Increased } \\
\text { ADE }\end{array}$ \\
\hline
\end{tabular}

Sin and McAlister ${ }^{(5)}$ evaluated in retrospective fashion a cohort of 1162 patients treated with beta blockers for heart failure. An evaluation of ACE inhibitor therapy was performed as a secondary analysis. After controlling for age, sex, Charlson score, hypertension, and ischemic heart disease, beta blocker therapy was associated with a hazard ratio of $0.72(0.65-0.80)$ for all cause mortality, and 0.82 (0.74-0.92) for heart failure hospitalizations. The effect was dose-related. Those receiving higher doses of beta blockade had statistically significant lower rates of all cause mortality and heart failure hospitalizations than those who received lower doses.

The benefits were extended to those with chronic obstructive pulmonary disease, diabetes, systolic blood pressures less than 100, and bradycardia at baseline. Of note, $22 \%$ of the population sampled had a Charlson Comorbidity Index of at least 2 . The systolic function of these patients was not stated. The most commonly used beta blockers were metroprolol, sotalol (which was excluded from the analysis), and atenolol.

\section{Ace Inhibitors}

In the study published by Sin and McAlister, ${ }^{(5)}$ analysis of patients taking ACE inhibitors was also undertaken. Four thousand nine hundred and eight patients (4908) were prescribed ACE inhibitors during specified study period. The overall reduction in all cause mortality was $0.59(0.55-0.62)$. The reduction in heart failure-related hospitalizations was 0.93 (0.87-1.00). A dose-dependent effect was also present with a hazard ratio of $0.67(0.61-0.72)$ for those taking lower doses of ACE inhibitors, and 0.55 for those taking higher doses. ${ }^{(7)}$

Ahmed et al. ${ }^{(30)}$ published a propensity analysis of 295 patients with systolic heart failure and a mean age of 78.5 years. The authors evaluated patients discharged from hospital with an indication for ACE inhibitors. Patients prescribed ACE inhibitors were compared to patients that had an indication to be on an ACE inhibitor and were not prescribed one. Adherence to an ACE inhibitor regimen was specifically documented by following prescriptions. Patients that should have been on an ACE inhibitor that were not prescribed one 
had a mortality hazard ratio of 1.47 (1.03-2.08). The mean survival rate of those not prescribed ACE inhibitors was $22 \%$, with a mean survival time of 627 days. The survival rate of those prescribed ACE inhibitors was $33 \%$, with a mean survival time of 829 days. ${ }^{(30)}$

In a previous study published by Ahmed et al., ${ }^{(31)} 1090$ patients with a mean age of 79.1, ACE inhibitor use and evaluation of LV ejection fraction were both associated with decreased mortality in a three-year follow-up period posthospitalization for heart failure. ACE inhibitors conferred a hazard ratio of $0.76(0.71-0.96)$. In this study, the relative proportions of patients with systolic and diastolic heart failure were not reported. Sixty-one percent of patients had died after the three-year follow-up period.

Luthi et al. ${ }^{(32)}$ performed a retrospective cohort evaluation of 621 patients with a mean age of 77.4 years. All of these patients had systolic heart failure. Patients were assessed on whether or not they had received ACE inhibitor therapy posthospitalization for heart failure. Compared to those who had received an ACE inhibitor, those who did not receive an ACE inhibitor had a hazard ratio of 1.63 for mortality. Even patients who were receiving lower-than-recommended doses of ACE inhibitors had a nonsignificant hazard ratio of $1.3(0.86-1.97)$.

Gambassi et al. ${ }^{(29)}$ performed a retrospective cohort study. They compared the effect of digoxin therapy versus ACE inhibitor therapy. A mortality benefit was noted; however, the interpretation of this trial was difficult because ACE inhibitor and digoxin therapy are not considered equivalent therapy in the general HF population. Elderly patients did have a mortality benefit if they took ACE inhibitors as opposed to Digoxin.

\section{CONCLUSION}

In retrospect, there is a relative paucity of high quality studies to guide heart failure therapy in the elderly. The conclusions drawn from this study must be taken with caution because of the reliance on data from smaller observational studies and many retrospective studies. Perhaps the most important conclusion is that there is more work that needs to be done to characterize ADEs in the elderly, understand the magnitude of the effect size of these medications on morbidity and mortality, and understand whether the potential side effects outweigh the benefits in a quality-adjusted life years analysis.

Despite the limitations, the data in this review seems to suggest that there is an increased adverse drug event rate in relation to beta blocker and spironolactone therapy. The increased adverse drug event rate was noted in observational studies, population-based studies, and randomized control trials. In the case of beta blockers, specifically increased amounts of bradycardia, hypotension, and syncope were noted. ${ }^{(12,13,14,15,16,17,18)}$

Studies on spironolactone revealed increased rates of hyperkalemia, acute renal failure, and medication discontinuation, although study populations were small. ${ }^{(23,24,25)}$
Given the mere suggestion that the older persons with heart failure experience adverse events to a greater degree, we feel that further characterization in prospective studies is warranted.

It is possible that the less rigorous follow-up that patients receive outside of randomized controlled trials could contribute to the increased rates of ADEs. It remains unclear, however, how age and frailty could be independent predictors. The increased events rates in both the RCT and observational data when compared to younger patients do beg that question.

With regards to efficacy, it appears that the benefits of beta blockers and ACE inhibitors extend to the very elderly heart failure population. This trend was noted in both largescale retrospective analyses, as well as a prospective blinded randomized control trial in the case of beta blockers. ${ }^{(5,18,19)}$ The effect appears to apply to both hospitalizations and mortality; however; the magnitude of the effect size was not equal across all studies.

In the SENIORS trial, the magnitude of the effect size was smaller than it was in the younger patient populations, and statistical significance was not gained when measuring mortality as an endpoint. It is possible that the class effect shared between metroprolol, carvedilol, and bisoprolol for systolic heart failure does not translate directly to the use of nebivolol. Another possibility, which the study seemed to suggest, is that the population greater than 75 did not benefit as much from the intervention and skewed the results in a negative fashion. The patients under 75 did benefit in a similar magnitude to other heart failure studies with similar relative risk reductions. ${ }^{(18)}$

Despite the fact that the elderly do have multiple medical comorbidities and that they were measured in many studies, there are no clear predictors of adverse events based on a validated prediction model. For beta blockers, advanced NYHA class and decreased left ventricular ejection fraction predicted poor tolerability in two studies. Possible predictors include advanced age, obstructive airways disease, and baseline blood pressure, although the data were conflicting. Further evaluation is necessary. Functional status was measured but not utilized in predicting outcomes with any HF therapy. ${ }^{(13,17,23)}$

Additional information that would be valuable in this patient population is the measurement of quality-adjusted life years. Patients may survive longer and avoid hospitalization, but if the side effects that they experience severely diminish the quality of the life that they experience, perhaps the therapy is not as valuable in this patient population as it clearly is in patients with a mean age of approximately 65 .

In the study by Sin and McCalister, ${ }^{(5)}$ the mortality benefits were seen despite controlling for adverse events such as bradycardia. Despite that fact, perhaps living longer while experiencing significant side effects or functional impairment imposed by these medications is not what these patients desire.

There were several limitations in our study. The first is the strong dependence upon observational data due to paucity of data from randomized studies. In spite of this, the 
observational studies, specifically related to the efficacy of beta blocker and ACE inhibitor therapies, were very large and of higher quality. ${ }^{(5,18)}$ Perhaps this allows for stronger external validity for the conclusions made with regard to these topics. We did attempt to control for heterogeneity where possible, but even this methodology has its limitations in terms of minimizing bias.

The spironolactone studies were small in number, and utilized smaller populations. Also, we attempted to extrapolate answers from studies that the original design of the study was not constructed to answer. Specifically, the focus of the Juurlink study was not to relate increased rates of adverse events to age, but to the application of RCT data to "real-life" circumstances. However, the fact that the findings of increased ADEs in an older population did parallel the findings of the observational data strengthens the overall conclusions made. ${ }^{(23,24,25)}$

The second limitation is the nonuniform group of patients involved. ACE inhibitor, beta blocker therapy, spironolactone, and digoxin are indicated for the reduction of morbidity and mortality for systolic heart failure only. Some of the observational studies utilized did not distinguish between the two.

Patients with diastolic heart failure are sometimes mistakenly treated with systolic HF medications. Diastolic HF is extremely common in the elderly. We felt that it was important to include the diastolic heart failure patients in order to detect if there were significant number of diastolic heart failure patients that were being inappropriately treated and being exposed to side effects without any hope of benefiting from the medications. We did not find this explicitly in this study. However, including these patients increased the heterogeneity of our study. Where possible, the distinction between the two has been made in the manuscript.

Our study points to the need for randomized control trials for systolic heart failure in the elderly, specifically with the medications that are already known to be efficacious in younger populations. The adverse event rates need to be measured in a comprehensive manner. The standardized measurement of medical comorbidities, functional status, and quality of life are essential. Finally, the above information should be analyzed to see whether or not prediction rules exist for adverse events and tolerability.

\section{CONFLICT OF INTEREST DISCLOSURES}

The authors declare that no conflicts of interest exist.

\section{REFERENCES}

1. Cowie MR, Fox KF, Wood DA, et al. Hospitalization of patients with heart failure. A population-based study. Eur Heart J. 2002;23(11):877-885.

2. Shibata MC, Flather MD, Wang D. Systematic review of the impact of beta blockers on mortality and hospital admissions in heart failure. Eur J of Heart Failure. 2001;3(3):351-357.
3. Senni M, Tribouilloy CM, Rodeheffer RJ, et al. Congestive heart failure in the community. A study of all incident cases in Olmsted County, Minnesota, in 1991. Circulation. 1998;98:2282-2289.

4. Philbin EF, Rocco RA, Lindenmuth NW, et al. Clinical outcomes in heart failure: report from a community hospital-based registry. Am J Med. 1999;107(6):549-555.

5. Sin D, McAlister F. The effects of beta-blockers on morbidity and mortality in a population-based cohort of 11,942 elderly patients with heart failure. Am J of Med. 2002;113(8):650-656.

6. Pitt B, Zannad F, Remme WJ, et al. The effect of spironolactone on morbidity and mortality in patients with severe heart failure. NEJM. 1999;341(10):709-717.

7. Lindeman RD, Tobin JD, Shock NW. Longitudinal studies on the rate of decline of renal function with age. J Am Geriatr Soc. 1985;33(4):278-285.

8. Weidmann P, De Myttenaere-Buesztein S, Maxwell MH., et al. Effect of aging on plasma renin and aldosterone in normal man. Kidney Int. 1975;8:325-333.

9. Noth RH, Lassman MN, Tan SY, et al. Age and the rennin-aldosterone system. Arch Intern Med. 1977;137(10):1414-1417.

10. Clark HD, Wells GA, Huëtt C, et al. Assessing the quality of randomized trials: reliability of the Jadad scale. Control Clinical Trials. 1999;20(5):448-452.

11. Wells GA, Shea B, O'Connell D, et al. The Newcastle-Ottawa Scale (NOS) for assessing the quality of nonrandomised studies in meta-analyses. Accessed from the internet January 4th, 2011: http://www.ohri.ca/programs/clinical_epidemiology/oxford.asp

12. Del Sindaco D, Pulignano G, Cioffi G, et al. Safety and efficacy of carvedilol in very elderly diabetic patients with heart failure. J Cardiovasc Med. 2007;8(9):675-682.

13. Krum H, Hill J, Fruhwald F, et al. Tolerability of beta-blockers in elderly patients with chronic heart failure: the COLA II study. Eur J Heart Failure. 2006;8(3):302-307.

14. Cioffi G, De Feo S, Pulignano G, et al. Does atril fibrillation in very elderly patients with chronic systolic heart failure limit the use of carvedilol? Int J Cardiol. 2006;107(2):220-224.

15. Lawless C, Tamlyn T, Shah R, et al. Titration of carvedilol in elderly heart failure patients. Am J Geriatr Cardiol. 2005;14(5):230-235.

16. Baxter AJ, Spensley A, Hildreth A, et al. Beta-blockers in older persons with heart failure: tolerability and impact on quality of life. Heart. 2002;88(6):611-614.

17. Witham M, Gillespie N, Struthers A. Age is not a significant risk factor for failed trial of beta-blocker therapy in older patients with chronic heart failure. Age Ageing. 2004;33(5):467-472.

18. Flather M, Shibata M, Coats A, et al. Randomized trial to determine the effect of nebivolol on mortality and cardiovascular hospital admission in elderly patients with heart failure. Eur Heart J. 2005;26(3):215-225.

19. Packer M, Fowler M, Roecker EB, et al. Effect of carvedilol on the morbidity of patients with severe chronic heart failure: results of the carvedilol prospective randomized cumulative survival (COPERNICUS) study. Circulation. 2002;106(17):2194-2199. 
20. Haffner CA, Kendall MJ, Struthers AD, et al. Effects of captopril and enalapril on renal function in elderly patients with chronic heart failure. Postgrad Med J. 1995;71(835):287-292.

21. Mets T, De Bock V, Praet JP. First-dose hypotension, ACE inhibitors, and heart failure in the elderly. Lancet. 1992;339(8807):1487-1488.

22. Zi M, Carmichael N, Lye M. The effect of quinapril on functional status of elderly patients with diastolic heart failure. Cardiovasc Drugs Ther. 2003;17(2):133-139.

23. Dinsdale C, Wani M, Steward J, et al. Tolerability of spironolactone as adjunctive treatment for heart failure in patients over 75 years of age. Age Ageing. 2005;34(4):395-398.

24. Juurlink DN, Mamdani M, Lee DS, et al. Rate of hyperkalemia after publication of the randomized aldactone evaluation study. NEJM. 2004;351(6):543-551.

25. Butler JV, McAvoy H, McEnroy D, et al. Spironolactone therapy in older patients - the impact of renal dysfunction. Arch Gerontol Geriatr. 2002;35(1):45-49.

26. Carosella L, Pahor M, Pedone C, et al. Digitalis in the treatment of heart failure in the elderly. The GIFA study results. Arch Gerontol Geriatr. 1996;23(3):299-311.

27. Misiaszek B, Heckman GA, Merali F, et al. Digoxin prescribing for heart failure in elderly residents of long-term care facilities. Can J Cardiol. 2005;21(3):281-286.
28. Heckman GA, Misiaszek B, Merali F, et al. Management of heart failure in Canadian long-term care facilities. Can J Cardiol. 2004;20(10):963-969.

29. Gambassi G, Lapane KL, Sgadari A, et al. Effects of angiotensin-converting enzyme inhibitors and digoxin on health outcomes of very old patients with heart failure. SAGE Study Group. Arch Internal Med. 2000;160(1):53-60.

30. Ahmed A, Centor RM, Weaver MT, et al. A propensity score analysis of the impact of angiotensin-converting enzyme inhibitors on long-term survival of older adults with heart failure and perceived contraindications. Am Heart J. 2005;149(4):737-743.

31. Ahmed A, Maisiak R, Allman RM, et al. Heart failure mortality among older medicare beneficiaries: association with left ventricular function evaluation and angiotensin-converting enzyme inhibitor use. Southern Med J. 2003;96(2):124-129.

32. Luthi JC, McClellan WM, Fitzgerald D, et al. Mortality associated with the quality of care of patients hospitalized with congestive heart failure. Int J Qual Health Care. 2002;14(1):15-24.

Correspondence to: Dr. Richard Sztramko, BHSc, MD, FRCPC, Division of Geriatrics, Vancouver General Hospital, Diamond Centre, 7th Floor, 2775 Laurel St., Vancouver, BC V5Z 1M9 Canada

Email: rich.sztramko@utoronto.ca 